\title{
Tuberculosis screening in pediatric patients receiving TNF- alpha inhibitor therapy
}

\author{
Manolya Acar ${ }^{1}$, Murat Sütçü${ }^{1}$, Hacer Aktürk ${ }^{1}$, Selda Hançerli-Torun ${ }^{1}$, Oğuz Bülent Erol², \\ Nuran Salman ${ }^{1}$, Ayper Somer ${ }^{1}$ \\ ${ }^{1}$ Division of Infectious Diseases, Department of Pediatrics, İstanbul University Istanbul Faculty of Medicine; ${ }^{2}$ Pediatric \\ Radiology, Private Clinic, Istanbul, Turkey. E-mail: manolya_kara@yahoo.com \\ Received: 9th February 2017, Revised: 25th April 2017, Accepted: 30th June 2017
}

\begin{abstract}
SUMMARY: Acar M, Sütçü M, Aktürk H, Hançerli-Torun S, Erol OB, Salman N, Somer A. Tuberculosis screening in pediatric patients receiving TNF-alpha inhibitor therapy. Turk J Pediatr 2017; 59: 503-510.

Tumor necrosis factor-alpha inhibitors (TNFIs) increase the risk of tuberculosis (TB). The aim of this study was to evaluate pediatric patients who are under TNFIs regarding the emergence of $\mathrm{TB}$, and to determine the effectiveness of screening methods. This was a retrospective observational study of 73 patients receiving TNFIs therapy for at least 6 months duration between January 2011 and January 2016. Detailed demographic and clinical data were gathered from patients' files. Seventy-three patients (female $n=41,56.2 \%$ ) with a median age of 129 (38-215) months were enrolled. Median follow-up period was 18 (6-60) months. Median duration of primary illness prior to TNFI therapy was $24(2-184)$ months. Sixteen patients $(21.9 \%)$ with latent tuberculosis infection (LTBI) were given isoniazid (INH) prior to TNFI therapy. TNFIs were adalimumab $(n=39,53.5 \%)$, infliximab $(n=22,30.1 \%)$ and etanercept $(\mathrm{n}=12,16.4 \%)$. During follow-up, 16 patients $(21.9 \%)$ were started on INH treatment. Median time of starting INH was 20 (4-42) months. One patient (3.1\%) who received INH had elevated liver transaminases. One patient (1.3\%) developed active TB during follow-up. In conclusion, patients who are candidates for TNFI treatment should be screened for TB before and during therapy.
\end{abstract}

Key words: TNF-alpha inhibitor, tuberculosis, screening.

After the introduction of tumor necrosis factor-alpha inhibitor (TNFI) drugs in clinical medicine, a considerable extent of improvement has been achieved for the treatment of autoimmune disorders. As its name indicates, TNFIs suppress the activity of TNF- $\alpha$, which is a mediator that can cause inflammation and lead to immune system disorders such as rheumatoid arthritis, ankylosing spondylitis and inflammatory bowel diseases. ${ }^{1}$ On the other hand, protective effect of TNF- $\alpha$ against mycobacterial infections has been shown in several studies. ${ }^{2,3}$ Once the mycobacteria enters the human body, it cannot be killed but stay dormant in granulomas. TNF- $\alpha$ has a critical role in the migration of the inflammatory cells to the granuloma and maintenance of its stabilization. ${ }^{4,5}$ Besides this, TNF- $\alpha$ together with interferon gamma, induce antimicrobial activity, conduct the mobility of leukocytes, facilitate the migration of leukocytes through endothelium and activate cytotoxic $\mathrm{T}$ cells. Adequate amount of TNF- $\alpha$ is the corner stone of a healthy granuloma. In the presence of decreased TNF- $\alpha$ levels, granulomas are disorganized with ineffectively activated macrophages and high microbial load. On the other hand, when TNF- $\alpha$ is in an excessive amount, macrophages are over stimulated, cells within the granuloma die and mycobacteria continue to grow extracellularly. ${ }^{6}$

Five TNFIs, etanercept, infliximab, adalimumab, certolizumab and golimumab have been approved for the treatment of selected rheumatologic 
diseases by the United States Food and Drug Administration (FDA). Among those, only etanercept is a soluble TNF receptor fusion protein and the latter are monoclonal antiTNF antibodies. Certolizumab and golimumab are newer agents and there are limited data regarding side effects.

TNFIs increase the reactivation risk of latent tuberculosis infection (LTBI). It is not unexpected since TNF- $\alpha$ has crucial roles on pathogenesis of tuberculosis (TB). According to the FDA adverse event reporting system, the estimated incidence of TB among the patients on TNAI treatment was 54 and 28/100,000 for etanercept and infliximab, respectively, in United States between1998-2002. This rate was higher than the actual TB incidence of the country $(5.2-6.8 / 100,000)$ at the same period of time. ${ }^{7}$ For this reason, Centers for Disease Control and Prevention (CDC) recommends LTBI screening of all patients prior and during TNFI therapy. ${ }^{8}$

In 2014, World Health Organization estimated the TB incidence as 18 (16-21) per 100,000 population in Turkey. ${ }^{9}$ In addition to reactivation of LTBI, new infection risk is also considerably high for our country. Therefore, it is particularly important for us to screen our patients who are candidates for TNFI therapy in terms of tuberculosis. A national guideline regarding TB management for patients who are using TNFI drugs has been published recently. ${ }^{10}$ In this manner, a rational approach for these patients can be executed. With the aid of this guideline, further studies will clarify this unclear era. The aim of this study was to evaluate pediatric patients who are under TNFI drugs regarding the emergence of $\mathrm{TB}$, and to determine the effectiveness of screening methods.

\section{Material and Methods}

This was a retrospective observational study evaluating the risk of TB on patients receiving TNFI therapy at the Pediatric Infectious Disease Clinic of Istanbul University Faculty of Medicine between January 2011 and January 2016. Seventy-three patients, who had been given TNFI therapy for at least 6 months duration were included in the study. Detailed data including demographics of the patients, primary disease requiring TNFI treatment, the duration of primary disease, duration of TNFIs, prior and concomitant medication history, presence of immune suppressive drug use, history of TB contact, presence and numbers of Bacillus Calmette-Guerin (BCG) scar, laboratory test results (complete blood count, liver transaminases, urea and creatinine levels, erythrocyte sedimentation rate and C-reactive protein values), radiological examination reports evaluated by pediatric radiologists including chest $\mathrm{X}$ ray and/or computerized thorax tomography was gathered from patients' charts.

In our clinic, patients who are candidates for TNFI therapy have been routinely screened with detailed medical history, physical examination, tuberculin skin test (TST) and/or interferon gamma release assay (IGRA) and chest $\mathrm{x}$-ray prior to therapy as it is recommended. ${ }^{10}$

Before the publication of the mentioned guideline our clinical approach was to reevaluate those patients every three months with history, physical examination and chest X-ray together with TST (twice a year) and microbiologic investigations when needed.

TST was performed on the 2/3 upper volar surface of the forearm using Mantoux method in which five tuberculin test units of purified protein derivative was injected intradermal and the result was recorded as the transverse diameter in millimeters of induration at $72 \mathrm{~h}$. At initial evaluation, in patients who had never been given immunosuppressive therapy, TST $\geq 15 \mathrm{~mm}$ (if BCG vaccinated) and TST $\geq 10 \mathrm{~mm}$ (if BCG unvaccinated) were considered as positive. In the case of prior immunosuppressive drug use TST cut-off limit was accepted as $5 \mathrm{~mm}$.

IGRA was performed by QuantiFERON ${ }^{\circledR}$-TB Gold In-Tube (QFT-GIT) test. For QFT-GIT test, $3 \mathrm{ml}$ peripheral blood was taken from each patient included in the study and $1 \mathrm{ml}$ added to each of three tubes in the QFT-GIT test kit (nil, TB antigen, and mitogen) and the tubes were shaken for approximately $5 \mathrm{~s}$. The tubes were first incubated at $37^{\circ} \mathrm{C}$ for $16-24 \mathrm{~h}$. After the incubation, the tubes were centrifuged at $2500 \times \mathrm{g}$ for $5 \mathrm{~min}$ to separate plasma, and the separated plasma were transferred into $1.5 \mathrm{ml}$ microcentrifuge tubes (Eppendorf) and stored at $-20^{\circ} \mathrm{C}$ until ELISA.

Patients were considered to have LTBI if either 
TST and/or IGRA was positive, or evidence of LTBI such as fibrotic changes, calcification areas larger than $5 \mathrm{~mm}$, pleural thickening and linear opacities were observed in chest radiography. Patients with LTBI were given isoniazid (INH) treatment $(10 \mathrm{mg} / \mathrm{kg} /$ dose, $\max 300 \mathrm{mg}$, once daily) at least one month prior and were continued to have it for a total of 9 months.

Statistical analysis of data was performed with statistical package for social science (SPSS) for Windows version 21.0 (SPSS 21.0, SPSS Inc. USA). Categorical and numerical variables between groups were compared by using Fisher Chi-square and independent sample $t$ tests respectively. Statistical significance limit was accepted as $\mathrm{p}<0.05$.

This study was performed with the permission of Istanbul University Faculty of Medicine Clinical Research Ethical Committee.

\section{Results}

A total number of 73 patients (female $n=41$, $56.2 \%)$ with a median age of 129 (38-215) months were enrolled in the study. Clinical characteristics of patients on initial evaluation are summarized in Table I. Median follow-up period was 18 (6-60) months.

All of the patients had chest X-ray initially. Among those, nonspecific consolidation $(n=2$, $2.8 \%)$ and nodular opacification $(\mathrm{n}=1,1.4 \%)$ were obtained and reevaluated by CT. Neither of these were compatible with TB. Sixteen patients $(21.9 \%)$ with LTBI were given INH prior to TNFI therapy. Isoniazid had been administered at least one month before TNFIs and was continued for 9 months totally.

During our follow-up period, 16 patients (21.9\%) were started on INH treatment. These patients had negative TSTs initially which became $\geq 5 \mathrm{~mm}$ during TNFI treatment. Characteristics of these patients are summarized in Table II. Median time of starting INH treatment was 20 (4-42) months. Among those, the most common preferred agent was adalimumab $(n=10,62.5 \%)$. Two patients (12.5\%) had etanercept and 4 patients (25\%) had infliximab therapy. Primary diagnosis of these patients were unspecified uveitis $(n=8$, $50 \%)$, inflammatory bowel disease $(n=2$, $12.5 \%)$, psoriasis $(n=1,6.2 \%)$ and sarcoidosis $(\mathrm{n}=1,6.2 \%)$. Median duration of primary illnesses prior to INH therapy was 32 (6-120) months. There was no statistical difference among different agents and primary illnesses in terms of TB risk ( $\mathrm{p} 0.91$ and 0.35 , respectively). Only one of the patients $(3.1 \%)$ who received INH had elevated liver transaminases which returned to normal levels after a reduction in INH dose. It showed no more increase after tapering normal dosage.

One patient $(1.3 \%)$, a-12-year-old boy with the diagnosis of juvenile idiopathic arthritis, developed active TB during follow-up. He had been given systemic corticosteroids and methotrexate for three years prior to antiTNF-alpha treatment and he was started on adalimumab after that. His TST was negative
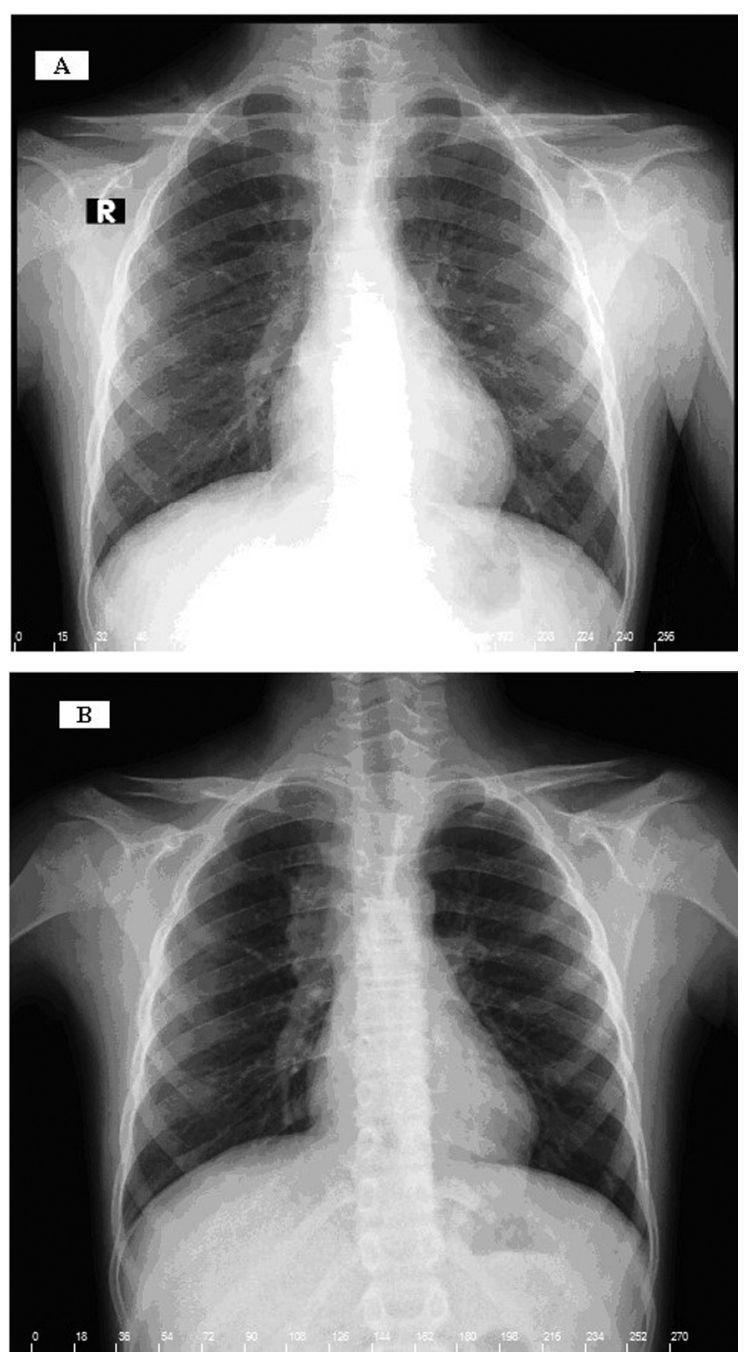

Fig. 1. Chest X-ray findings. A. Normal chest X-ray, initially. B. Paratracheal opacity on the third month of the therapy. 
and chest X-ray was normal initially (Fig. 1a). On third month of his therapy, his father was diagnosed as pulmonary TB and he was started on INH. Besides, adalimumab was terminated. One month later, his TST test was measured as $7 \mathrm{~mm}$ and chest X-ray revealed paratracheal opacity (Fig. 1b). Thorax CT confirmed a necrotic mass lesion with diameter of $40 \times 27 \times 24 \mathrm{~mm}$ which is compatible with tuberculosis (Figure-2). He was started on antituberculous therapy including isoniazid, rifampicin, pyrazinamide and ethambutol.
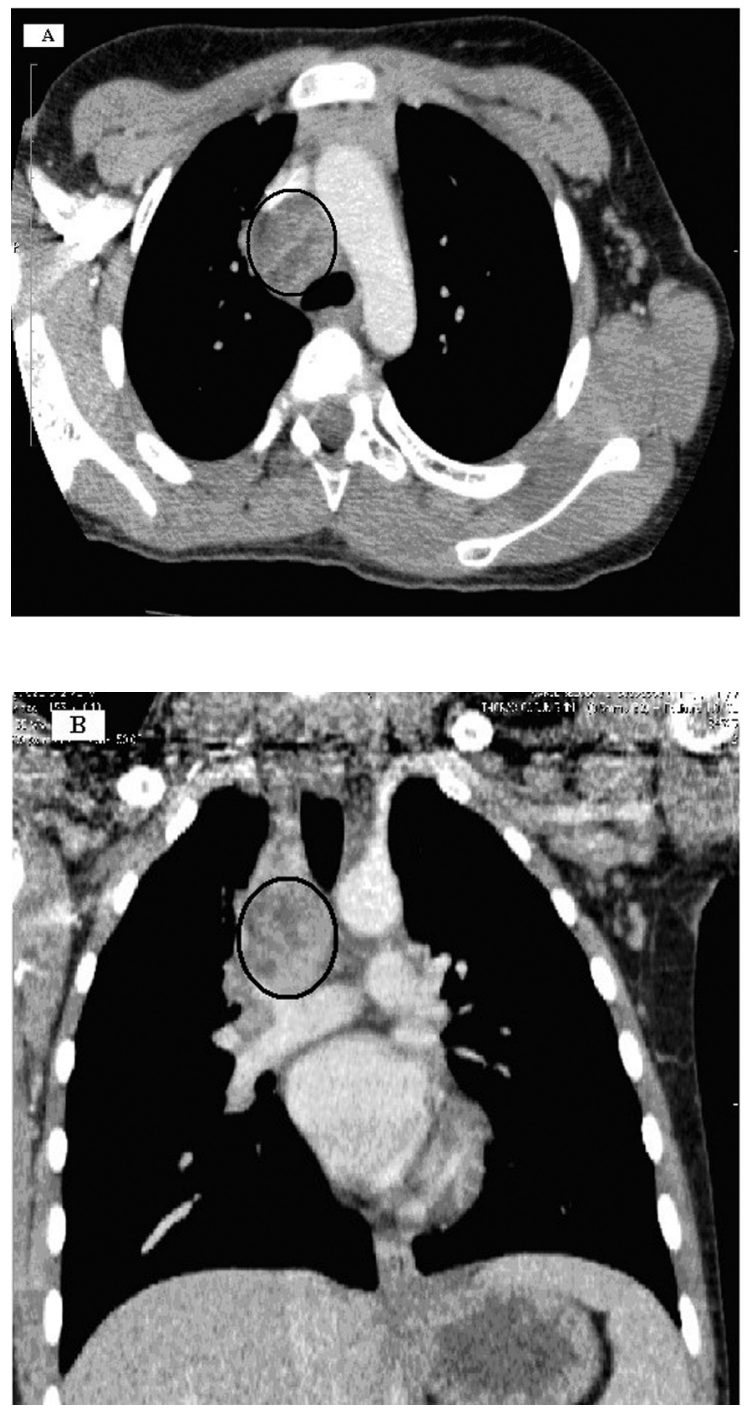

Fig. 2. Thorax CT findings. Mass lesion $40 \mathrm{mmx} 27 \mathrm{mmx}$ $24 \mathrm{~mm}$ in diameter extending from right upper paratracheal region to lower paratracheal region and right hilus A. Transverse section B. Sagittal section.

\section{Discussion}

TNF-alpha has a crucial role in the host response to a variety of infections, particularly those involving Mycobacterium tuberculosis and other intracellular pathogens. Therefore, the risk of tuberculosis, both new infection and reactivation of LTBI, increases with the use of TNFIs. However, the risk of reactivation $\mathrm{TB}$ is not virtually the same for all TNFIs. A study from United Kingdom, reported that the incidence of TB among patients receiving TNFI therapy, was the highest for adalimumab $(144 / 100,000)$ followed in the frequency by infliximab $(136 / 100,000)$ and etanercept $(39 / 100,000) .{ }^{11}$ There are several theories trying to explain this difference. First of them is the pharmacokinetic hypothesis. It is postulated that, intravenous administration of infliximab increases blood drug levels very quickly unlike etanercept, which is applied subcutaneously. However, although adalimumab has similar pharmacokinetic properties to etanercept, the risk with the former is the highest. ${ }^{12}$ Ringheanu et al. ${ }^{13}$ reported decreased interferon gamma production and CD4 cell response against $\mathrm{TB}$ with adalimumab and infliximab use. Such response was not observed with etanercept therapy. In addition, these agents have different sites of action. While adalimumab and infliximab directly inhibit TNF- $\alpha$, etanercept binds to TNF- $\alpha$ receptors abbreviated as TNF-R1 and TNF-R2. Among those, TNF-R1 has the main role in granuloma formation which etanercept has lower affinity. ${ }^{1}$ Presumably, all of these factors contribute to this difference. In our study, although the difference was not statistically significant, most of the patients who had been started isoniazid were receiving adalimumab therapy $(n=10$, $62.5 \%$ ). Furthermore, our active tuberculosis case was also receiving adalimumab.

Tuberculosis cases that occur in a short period of time after the initiation of TNFIs are most commonly reactivation TB. Whereas those that occur later may represent either delayed reactivation or newly acquired $\mathrm{TB}$ infection. ${ }^{14}$ However, this discrimination can only be made by DNA typing method which is required especially in places with high TB burden, like our country. Besides, time required to TB onset also differs among different TNFIs. Keane et al. ${ }^{13}$ reported that time to TB onset was the 
Table I. Clinical Data of Patients on Initial Evaluation.

Female (n,\%)

$41(56.2)$

Age (months), median (range)

$129(38-215)$

Follow-up period (months), median (range)

$18(6-60)$

Primary disease, $\mathrm{n}(\%)$

Unspecified uveitis

Juvenile idiopathic arthritis

$39(53.4)$

$16(21.9)$

8 (11)

$4(5.5)$

Psoriasis

$3(4.1)$

Sarcoidosis

$3(4.1)$

Behcet's disease

$24(2-184)$

Duration of primary disease (months), median (range)

Prior immune suppressive drug use, $\mathrm{n}(\%)$

Systemic corticosteroids

$60(82.2)$

Methotrexate

$47(64.4)$

Cyclophosphamide

19 (26)

Azathioprine

$11(15.1)$

Mycophenolate mofetil

$1(1.4)$

$2(2.8)$

$4(5.5)$

Positive history of tuberculosis contact, $\mathrm{n}(\%)$

Tuberculin skin test, $\mathrm{n}(\%)$

$0 \mathrm{~mm}$

$44(60.2)$

$0-4 \mathrm{~mm}$

$11(15.1)$

$\geq 5 \mathrm{~mm}$

$18(24.7)$

IGRA (number of patients performed), $\mathrm{n}(\%)$

Positive

Negative

Undetermined

$3(4.1)$

$54(74)$

0

BCG scar, n(\%)

No

$7(9.6)$

$\geq 1$

$66(90.4)$

TNFI therapy, $\mathrm{n}(\%)$

Etanercept

$12(16.4)$

Infliximab

$22(30.1)$

Adalimumab

39 (53.5)

Concomitant treatment, $\mathrm{n}(\%)$

Systemic corticosteroids

45 (61.6)

Methotrexate

$37(50.7)$

Cyclophosphamide

$13(17.8)$

Azathioprine

$9(12.3)$

None

$5(6.8)$

$14(6-72)$ 


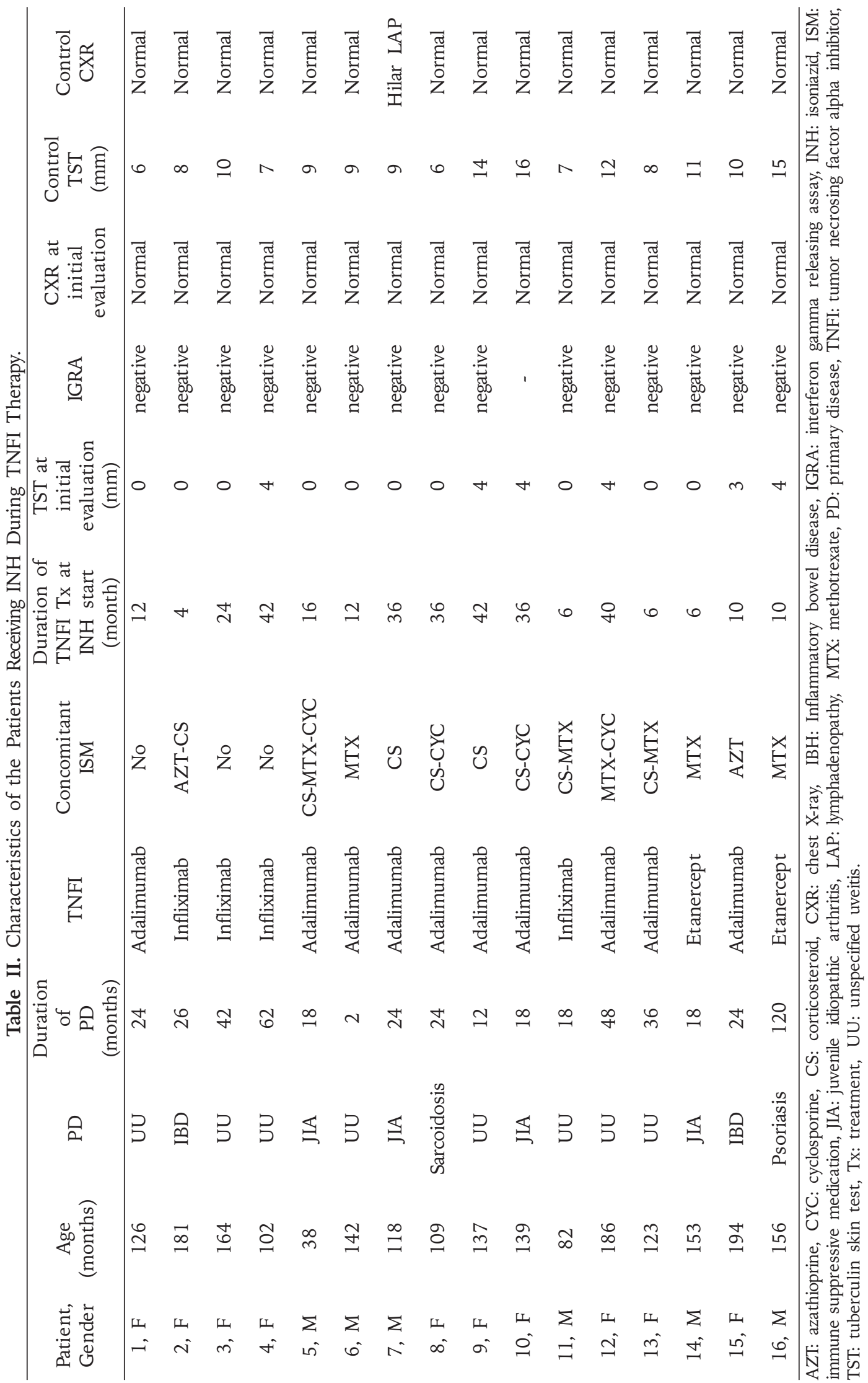


shortest for infliximab (5.5 months) when compared to etanercept (13.4 months) and adalimumab (18.5 months). In an earlier study from our country, in which a total of 36 patients under etanercept treatment were evaluated for a median period of 11.5 months (3-48 months), no active TB case was observed. ${ }^{15}$ In our active $\mathrm{TB}$ case, time to $\mathrm{TB}$ onset was three months. But since the initial evaluation for TB was negative and his father had been diagnosed as pulmonary TB at the same time, possibility of reactivation TB is excluded. In addition, the other household contacts revealed negative screening results. For this reason, it is possible to explain our case as a newly acquired TB event that had occurred secondary to facilitator effect of TNFI agent.

According to CDC, LTBI screening with detailed history, physical examination, TST or IGRA of all patients who are candidates for TNFI drugs prior to therapy is recommended. Chest X-ray is indicated only if there is increased suspicion of TB with the mentioned screening methods ${ }^{8}$. However, since TB incidence is considerably high for our country, chest X-ray is routinely recommended in our national guidelines. ${ }^{10}$ Similarly, CDC does not recommend TST and IGRA combination for all patients. The advantage of IGRA over TST is the property of that it is not affected by BCG and can be helpful in the evaluation of BCG vaccinated TSTpositive patients. It is particularly important for our country since BCG vaccination is routinely performed at 2 months of age. In addition, IGRA is less influenced by immunosuppressive medications when compared to TST. ${ }^{16-18}$ On the other hand, IGRAs are expensive and require specialized laboratories. In developing countries like ours, cost effective approach should also be considered. Cobanoglu et al. ${ }^{19}$ found poor agreement between TST and the IGRA among 68 patients with chronic inflammatory diseases evaluated for LTBI before the use of TNFIs. In our study, IGRA was performed in $78.1 \%$ of our patients in which three of them revealed positive results. Among those, only one patient had negative TST and positive IGRA. So, IGRA did not show any superiority over TST in our study. In our active TB case, we were not able to perform IGRA due to financial reasons. His TST, which had been negative initially, became positive in correlation with the occurrence of the disease. Based on these findings, we can say that TST in the absence of IGRAs is still a good screening method.

In our study, a total number of 32 patients $(43.8 \%)$ were given INH treatment. Half of them had been started prior to TNFI therapy, while the other half was started during followup. This ratio is higher than another pediatric study reported by Kilic et al. ${ }^{20}$ from our country. In that study, $14.5 \%$ of the patients had been diagnosed as LTBI initially and $4.8 \%$ were started on INH during follow-up. This may be attributable to their approach of taking TST cut-off limit as $10 \mathrm{~mm}$. On the other hand, Cagatay et al. ${ }^{21}$ reported the chemoprophylaxis ratio as high as $83 \%$ in their study which had evaluated 702 patients. The TST cut-off limit was also $5 \mathrm{~mm}$ in that study, and the incidence of active TB was $0.85 \%$.

The main concern underlying increased chemoprophylaxis ratios are the risk of drug related side effects, mainly hepatotoxicity. In a study by Mutlu et al. ${ }^{22}$, among the patients who had been given chemoprophylaxis, the ratio of hepatotoxicity was $17.3 \%$. Approximately $5 \%$ of all patients interrupted INH therapy due to progressive hepatotoxicity. In the same study, the age of patients with hepatotoxicity was higher than the others. Although, a considerably high amount of patients had been given INH treatment, only one patient $(1.3 \%)$ had elevated liver enzymes who he did not require the cessation of the treatment. As none of our patients with LTBI presented with active TB, we can suggest that our protective approach is rational. The only active TB patient in our study was a newly acquired event.

Another concerning issue, especially for pediatricians is the radiologic toxicity of the children gained during screening period. Overuse of radiologic modalities can cause serious side effects. In our study, we performed chest CT in 4 patients $(5.4 \%)$ including the active TB case as we had the opportunity to collaborate with pediatric radiology clinic. This ratio is acceptable in this high risk patient group. Besides, in our active TB case, chest X-ray findings were also very directive. Therefore, it would be appropriate to evaluate chest X-rays with a specialist if possible. This approach will reduce the overuse of computerized tomography.

Once active TB is diagnosed in a patient 
receiving a TNFI agent, anti-TNF alpha therapy should be terminated. ${ }^{8,10,23}$ Meanwhile anti tuberculosis therapy should be initiated immediately. Although, some authors claim that TNFIs may be restarted after the clinical improvement is achieved, it would be proper to evaluate other treatment options. ${ }^{24} \mathrm{We}$ terminated TNFI agent immediately in our patient and although clinical improvement has been observed rapidly, we did not restart anti-TNF alpha therapy.

In conclusion, TB screening during TNFI therapy is mandatory. Detailed history, physical examination TST together with a well analyzed chest X-ray (2-4 times a year) would be the proper screening methods. It seems rational to accept TST cut-off limit as $5 \mathrm{~mm}$. Finally, the clinician should have a low threshold for treating such patients with given the increased risk emerging $\mathrm{TB}$ in patients receiving TNFIs.

\section{REFERENCES}

1. Schouwenburg PA, Rispens T, Wolbink GJ. Immunogenicity of anti-TNF biologic therapies for rheumatoid arthritis. Nat Rev Rheumatol 2013; 9: 164-172.

2. Flynn JL, Goldstein MM, Chan J, et al. Tumor necrosis factor-alpha is required in the protective immune response against Mycobacterium tuberculosis in mice. Immunity 1995; 2: 561-72.

3. Benini J, Ehlers EM, Ehlers S. Different types of pulmonary granuloma necrosis in immunocompetent vs. TNFRp55-gene-deficient mice aerogenically infected with highly virulent Mycobacterium avium. J Pathol 1999; 189: 127-137.

4. Bopst M, Garcia I, Guler R, et al. Differential effects of TNF and LTalpha in the host defense against M. bovis BCG. Eur J Immunol 2001; 31: 1935-1943.

5. Randhawa PS. Lymphocyte subsets in granulomas of human tuberculosis: An in situ immunofluorescence study using monoclonal antibodies. Pathology 1990; 22: $153-155$

6. Dorhoi A, Kaufmann SH. Tumor necrosis factor alpha in mycobacterial infection. Semin Immunol 2014; 26 : 203-209.

7. Wallis RS, Broder MS, Wong JY, et al. Granulomatous infectious diseases associated with tumor necrosis factor antagonists. Clin Infect Dis 2004; 38: 1261-1265.

8. Centers for Disease Control and Prevention (CDC). Tuberculosis associated with blocking agents against tumor necrosis factor-alpha--California, 2002-2003. MMWR Morb Mortal Wkly Rep 2004; 53: 683.

9. Estimates of TB and MDR-TB burden are produced by WHO in consultation with countries. Available at: www.who.int/tb/data. (Accessed May 10, 2016)
10. T.C Sağlık Bakanlığı Türkiye Halk Sağlığı Kurumu AntiTNF Kullanan Hastalarda Tüberküloz Rehberi. Available at: http://www.mhsm.gov.tr/images/diger_haberler/ anti_tnf_kullanan_hasta_tb_rehber.pdf. (Accessed December 12, 2016).

11. Dixon WG, Hyrich KL, Watson KD, et al. Drug-specific risk of tuberculosis in patients with rheumatoid arthritis treated with anti-TNF therapy: results from the British Society for Rheumatology Biologics Register (BSRBR). Ann Rheum Dis 2010; 69: 522-528.

12. Nestorov I. Clinical pharmacokinetics of TNF antagonists: How do they differ? Semin Arthritis Rheum 2005; 34: 12-18.

13. Keane J, Gershon S, Wise RP, et al. Tuberculosis associated with infliximab, a tumor necrosis factor alpha-neutralizing agent. N Engl J Med 2001; 345: 1098-1104.

14. Wallis RS. Mathematical modeling of the cause of tuberculosis during tumor necrosis factor blockade. Arthritis Rheum 2008; 58: 947-952.

15. Ayaz NA, Demirkaya E, Bilginer Y, et al. Preventing tuberculosis in children receiving anti-TNF treatment. Clin Rheumatol 2010; 29: 389-392.

16. Chapman AL, Munkanta M, Wilkinson KA, et al. Rapid detection of active and latent tuberculosis infection in HIV-positive individuals by enumeration of Mycobacterium tuberculosis-specific T cells. AIDS 2002; 16: 2285-2293.

17. Mow WS, Abreu-Martin MT, Papadakis KA, Pitchon HE, Targan SR, Vasiliauskas EA. High incidence of anergy in inflammatory bowel disease patients limits the usefulness of PPD screening before infliximab therapy. Clin Gastroenterol Hepatol 2004; 2: 309-313.

18. Schoepfer AM, Flogerzi B, Fallegger S, et al. Comparison of interferon-gamma release assay versus tuberculin skin test for tuberculosis screening in inflammatory bowel disease. Am J Gastroenterol 2008; 103: 27992806.

19. Cobanoglu N, Ozcelik U, Kalyoncu U, et al. Interferongamma assays for the diagnosis of tuberculosis infection before using tumour necrosis factor-alpha blockers. Int J Tuberc Lung Dis 2007; 11: 1177-1182.

20. Kilic O, Kasapcopur O, Camcioglu Y, et al. Is it safe to use anti-TNF-agents for tuberculosis in children suffering with chronic rheumatic disease? Rheumatol Int 2012; 32: 2675-2679.

21. Cagatay T, Aydan M, Sunmez S, et al. Follow-up results of 702 patients receiving tumor necrosis factor-alpha antagonists and evaluation of risk of tuberculosis. Rheumatol Int 2010; 30: 1459-1463.

22. Mutlu P, Sevinç C, Kılınç O, Uçan ES. Anti-TNF-alfa tedavisi verilen olgularda latent tüberküloz infeksiyonu açısından izlem sonuçlarımız. Nobel Medicus 2014; 10: $47-52$.

23. Shim TS. Diagnosis and treatment of latent tuberculosis infection due to initiation of Anti-TNF therapy. Tuberc Respir Dis 2014; 76: 261-268.

24. Matsumoto T, Tanaka T, Kawase I. Infliximab for rheumatoid arthritis in a patient; with tuberculosis. N Engl J Med 2006; 355: 740-741. 